\title{
Gauge-free cluster variational method by maximal messages and moment matching
}

\author{
Eduardo Domínguez, ${ }^{1,2}$ Alejandro Lage-Castellanos, ${ }^{1,2,3}$ Roberto Mulet, ${ }^{1,2}$ and Federico Ricci-Tersenghi ${ }^{4}$ \\ ${ }^{1}$ Department of Theoretical Physics, Physics Faculty, University of Havana, La Habana, CP 10400, Cuba \\ 2 "Henri Poincaré" group of Complex Systems, University of Havana, Cuba \\ ${ }^{3}$ CNRS, Laboratoire de Physique Statistique, École Normale Suprieure, 75005, Paris \\ ${ }^{4}$ Dipartimento di Fisica, INFN-Sezione di Roma 1 and CNR-Nanotec, unità di Roma, Università La Sapienza, P.le A. Moro 5, \\ 00185 Roma, Italy
}

(Received 24 November 2016; published 25 April 2017)

\begin{abstract}
We present an implementation of the cluster variational method (CVM) as a message passing algorithm. The kind of message passing algorithm used for CVM, usually named generalized belief propagation (GBP), is a generalization of the belief propagation algorithm in the same way that CVM is a generalization of the Bethe approximation for estimating the partition function. However, the connection between fixed points of GBP and the extremal points of the CVM free energy is usually not a one-to-one correspondence because of the existence of a gauge transformation involving the GBP messages. Our contribution is twofold. First, we propose a way of defining messages (fields) in a generic CVM approximation, such that messages arrive on a given region from all its ancestors, and not only from its direct parents, as in the standard parent-to-child GBP. We call this approach maximal messages. Second, we focus on the case of binary variables, reinterpreting the messages as fields enforcing the consistency between the moments of the local (marginal) probability distributions. We provide a precise rule to enforce all consistencies, avoiding any redundancy, that would otherwise lead to a gauge transformation on the messages. This moment matching method is gauge free, i.e., it guarantees that the resulting GBP is not gauge invariant. We apply our maximal messages and moment matching GBP to obtain an analytical expression for the critical temperature of the Ising model in general dimensions at the level of plaquette CVM. The values obtained outperform Bethe estimates, and are comparable with loop corrected belief propagation equations. The method allows for a straightforward generalization to disordered systems.
\end{abstract}

DOI: 10.1103/PhysRevE.95.043308

\section{INTRODUCTION}

The Ising ferromagnet is one of the most studied and celebrated models in statistical physics. Although it lacks a proper analytical solution in three dimensions, it is globally well understood [1]. However, the addition of disorder to this model generates a more complex scenario. Roughly speaking, the low temperature phase of the disordered model is not composed any more by two equivalent ordered phases as in the pure ferromagnetic model, but by many disordered phases with a complex structure. Techniques such as the replica trick [2] and the cavity method [3,4] opened the door to the analytical treatment of the disordered variants of this and similar models in fully connected or in locally treelike random graphs [5].

However, finite dimensional systems remain a challenging problem regarding the analytical solutions. Only recently [611] has it been realized that a proper generalization of the Bethe approximation, known with the name of cluster variational method (CVM), could be a good starting point for a systematic treatment of these kinds of disordered problems. The main task is to translate the (approximate) free energy saddle point conditions in a set of message passing equations, that can be solved efficiently even on large systems.

The interest in these kinds of approximations is not only theoretical, but it comes also from many applications outside physics. For example, in image processing [12-15], it is important to improve the quality of the reconstruction algorithms, and message passing derived from CVM approximations has proved to be a good candidate in this direction. Error correction and low density parity check (LDPC) codes is another example of applications where GBP has been studied [16], including the idea of fixing the gauge [17].

Most of previous works on cluster variational method and replica method, relied on the so-called parent-to-child message passing [6], which consists of an extension of the belief propagation for the Bethe approximation to more involved region graph approximations of the free energy. It has been shown that the parent-to-child message passing is redundant [17-19] since it introduces more "cavity" fields (messages) than actually needed, producing a sort of gauge invariance in the solution.

From a pure mathematical perspective, the gauge invariance is a consequence of solving a constrained optimization problem by introducing more Lagrange multipliers than actually needed. As a consequence, the dual problem (the one of extreme in terms of the multipliers) has many solutions (a continuous manifold), and some of the saddle point equations are redundant.

According to our experience, this invariance is not a big problem in the implementation of message passing algorithms on a given finite dimensional instance, but it certainly is a waste of computational resources since more parameters need to be implemented. In [20], however, authors report the gauge invariance as causing convergence problems, and in [19] authors remove the invariance in order to create a more robust message passing. In any case, this gauge invariance may obscure the connection between the average case prediction of the CVM equations derived for a disordered model and the solution of message passing equations in single instances of the model [8]. To alleviate this problem, we propose a general 
procedure to generate gauge-free generalized belief propagation (GFGBP) algorithm starting from a cluster variational method approximation.

The procedure developed consists of the following steps:

(1) definition of maximal messages, in place of parent-tochild messages;

(2) definition of moment matching fields in place of Lagrange multipliers to ensure beliefs consistency.

The first is just another possible choice of messages that is quite general. The latter is a change of perspective in the interpretation of messages as Lagrange multipliers forcing marginalizations, to fields forcing consistency of moments in the beliefs distributions. This allows a systematic construction of gauge-free message passing for any model with binary variables.

In $[17,18]$, authors developed a way to remove the redundancy in the GBP equations by removing the redundant messages. Our approach differs from theirs in that they keep with the parent-to-child approach of [6] and propose to fix the gauge by removing some messages completely from the belief expression of given regions in order to avoid loops in the region graph representation. We, instead, propose a larger set of messages, but with properly reduced degrees of freedom. While the idea of focusing on matching moments is not new, and is in the spirit of other message passing approximations, like the expectation consistent of [21] and even for the direct and inverse Potts model [22], here we use it as the fundamental argument to decide which degrees of freedom of messages can be discarded while still keeping a fully general CVM approximation.

We will apply the gauge-free approach to the computation of critical temperatures in the plaquette-CVM approximation in Ising model in general dimensions, obtaining analytical expressions that improve over Bethe. The high dimension expansion of the critical temperature is correct until the third order term, as is the loop calculus of Ref. [23]. We also test the procedure in single instance implementation of message passing in Ising model. The more complicated (and interesting) disordered models are left for future work.

The paper is organized as follows. Section II introduces CVM and message passing algorithm in general terms, while Sec. III explains the maximal messages (MM) and the moment matching (MM) approaches; finally, in Sec. IV we apply the MM-MM CVM (or 4M-CVM in short) algorithm to the calculation of the critical temperature in Ising models of general dimensions at the plaquette level. For the sake of readability, we defer to the appendixes the technical proofs.

\section{CLUSTER VARIATIONAL METHOD}

The kinds of problems we are dealing with are those statistical mechanics problems that require the computation of the properties of a large set of binary variables $x_{i} \in\{1,-1\}$, whose joint probability distribution

$$
P(\underline{x})=\frac{1}{Z} \exp [-\beta \mathcal{H}(\underline{x})]
$$

depends on a Hamiltonian $\mathcal{H}(\underline{x})$ that can be written as the sum of local terms

$$
\mathcal{H}(\underline{x})=\sum_{a} E_{a}\left(\underline{x}_{a}\right),
$$

where every interaction " $a$ " with energy $E_{a}\left(\underline{x}_{a}\right)$ involves a small subset of variables $\underline{x}_{a}$. This also includes the case of Bayesian networks, and therefore of many interesting inference problems.

Computations of the statistical properties of each variable $x_{i}$ or groups of them face the numerical difficulty of tracing over an exponential number of configurations when marginalizing over the remaining variables, and in general approximations are required. In the case of mean field, Bethe, and region graph approximations (see [24]), the underlying idea is to factorize the full probability distribution $P(\underline{x})$ into many smaller distributions containing a nonextensive number of variables that we will refer to as regions.

The CVM [6,25] starts from a set of maximal regions $R_{0}$ (basic clusters), where no region is subset of another, and constructs a hierarchy of regions over which the approximation is defined. We will require that each degree of freedom $x_{i}$ and also all interactions $E_{a}\left(\underline{x}_{a}\right)$ are present in at least one of these regions. Then, we extend $R_{0}$ with the closure under the intersection operation as explained next.

From $R_{0}$, we define recursively the set of intersections $R_{k}$ as

$$
R_{k}=\left\{r=r_{k-1} \cap r_{k-1}^{\prime} \mid r_{k-1}, r_{k-1}^{\prime} \in R_{k-1}\right\} .
$$

The whole group of regions is $R=R_{0} \cup R_{1} \cup R_{2} \ldots$ Actually, in the CVM construction, the same regions might appear more than once, and in different levels of intersections. Regardless this degeneracy, the relevant set is $R$, the set of all regions obtained, without paying attention to when or how many times they appear in the intersection process. Of utmost importance for later proofs is that $R$ is a closed set under intersections and a partially ordered set (poset), in which the subset relation defines the partial order, and $R_{0}$ is the set of maximal regions.

Since the system Hamiltonian is given by sums of local interactions between subsets of variables, we will consider that every time that the set of variables $\underline{x}_{a}$ are part of a given region $\underline{x}_{a} \subset r$, then the interaction " $a$ " itself is part of it, allowing us to define the energy of the region as

$$
E_{r}\left(\underline{x}_{r}\right)=\sum_{a \in r} E_{a}\left(\underline{x}_{a}\right) .
$$

Since all interactions are at least part of one maximal region $r_{0} \in R_{0}$, we can write the Hamiltonian of the system as a sum over regions:

$$
\mathcal{H}=\sum_{r \in R} c_{r} E_{r}\left(\underline{x}_{r}\right),
$$

where the counting numbers $c_{r}$ guarantee that every interaction is counted exactly once [6]:

$$
c_{\alpha}=1-\sum_{r \in A_{\alpha}} c_{r} .
$$




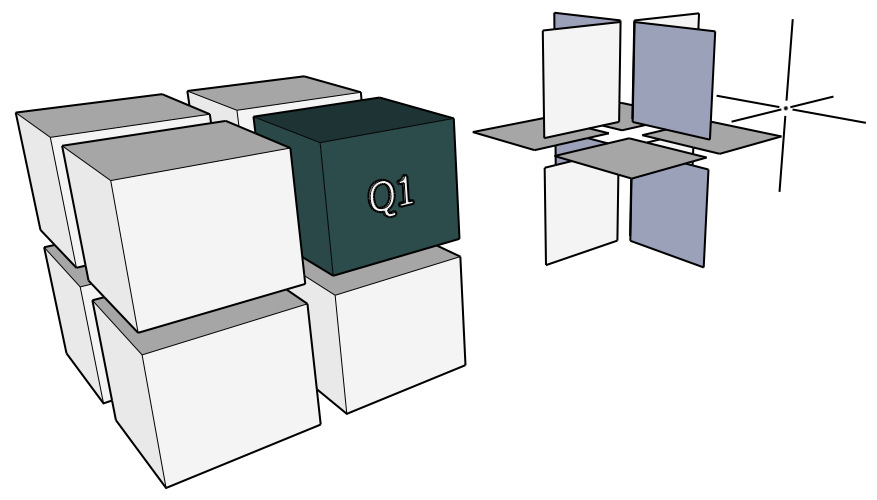

FIG. 1. Example of regions surrounding the central spin $s_{1}$ in the cube approximation for the 3D square lattice model. Left: the 8 cubic regions $Q_{1}, \ldots, Q_{8}$. Center: the 12 faces (plaquettes) $P_{1}, \ldots, P_{12}$ shared by the maximal cubic regions. Right: the 6 vertices shared by the plaquettes and the central spin. The cube $Q_{1}$ is highlighted for later use.

The set $A_{\alpha}$ stand for the set of all ancestors of region $\alpha$, this is all super-regions of region $\alpha$ :

$$
A_{\alpha}=\{r \in R \mid \alpha \subset r\} .
$$

Before going further in detail, let us visualize an example of the regions generated by the CVM construction. Consider a three-dimensional (3D) spin model, with spins living in the nodes of a 3D square lattice. In the cubic approximation, maximal regions are taken as the basic cubic cell of the lattice, with all its eight degrees of freedom at the cube's vertex. As a representative part of the full system, diagrams in Fig. 1 show all the regions containing the central spin $s_{1}$ (depicted as the central point in the rightmost diagram). Notice that the intersections of the cubic regions in the leftmost diagram produce the square plaquette regions in the center diagram, with a spin at every angle of the squares. And the intersection of those plaquettes results in the rod (edges with two spins) regions in the rightmost diagram, which intersect only in the central spin.

\section{A. Variational approach and message passing}

Next, we reproduce the approach by Zhou et al. [18] on the derivation of message passing equations, instead of that of Yedidia [6]. We prefer the former because it is somehow more direct in the choice of the belief equations, saving the time of passing through Lagrange multipliers.

It starts by noting that, in accordance with Eq. (2), the exact partition function of a system can be written as

$$
\begin{aligned}
Z(\beta) & \equiv \sum_{\underline{x}} \exp [-\beta \mathcal{H}(\underline{x})] \\
& =\sum_{\underline{x}} \prod_{r \in R}\left\{\exp \left[-\beta E_{r}\left(\underline{x}_{r}\right)\right]\right\}^{c_{r}} .
\end{aligned}
$$

A set of nonzero test functions $\left\{m_{z}\left(\underline{x}_{z}\right)\right\}$ can be multiplied and divided in the right hand side, such that they cancel out. We will call these test functions messages. Let us define $\partial z \subset R$ the set of regions in which the message $m_{z}\left(\underline{x}_{z}\right)$ appears, and let $D_{r}$ be the set of messages entering region $r$, then

$$
Z(\beta)=\sum_{\underline{x}} \prod_{r \in R}\left\{\exp \left[-\beta E_{r}\left(\underline{x}_{r}\right)\right] \prod_{z \in D_{r}} m_{z}\left(\underline{x}_{z}\right)\right\}^{c_{r}}
$$

will still be the same partition function (independently of the values of the messages) if

$$
\forall z \quad \sum_{r \in \partial z} c_{r}=0 .
$$

We can write an approximation to the free energy of the model in terms of the local beliefs

$$
b\left(\underline{x}_{r}\right)=\frac{1}{z_{r}} \exp \left[-\beta E_{r}\left(\underline{x}_{r}\right)\right] \prod_{z \in D_{r}} m_{z}\left(\underline{x}_{z}\right)
$$

with local partition functions

$$
z_{r}=\sum_{\underline{x}_{r}} \exp \left[-\beta E_{r}\left(\underline{x}_{r}\right)\right] \prod_{z \in D_{r}} m_{z}\left(\underline{x}_{z}\right) .
$$

The free energy of the model $F=-k T \ln Z(\beta)$ can be rewritten as

$$
\begin{aligned}
F & =-k T \sum_{r \in R} c_{r} \ln z_{r}-k T \ln \left[\sum_{\underline{x}} \prod_{r \in R} b_{r}\left(\underline{x}_{r}\right)^{c_{r}}\right] \\
& =F_{R}+\Delta F .
\end{aligned}
$$

This expression is still exact (independently of the value of the message functions). We will regard the first term $F_{R}[\{m\}]$ as a variational approximate to the real free energy. The rationale for this goes as follows. It can be shown (and will be) that the minimization of the first term is equivalent to imposing local consistency between marginals of the belief functions (those appearing in the second term). Once the beliefs are locally consistent, it can be shown that the correct joint probability distribution of the model $P(\underline{x})$ can be written in a factorized form as $\prod_{r \in R} b_{r}\left(\underline{x}_{r}\right)^{c_{r}}$ as far as the underlaying graph is a tree, therefore, $\Delta F=-K T \ln 1=0$. This proves, en passant, that the approximation is exact for the case of tree topologies. A rigorous justification of the approximation is absent, but in [10] authors relate $\Delta F$ to the sum of correction contributions in the loop expansion of the free energy. In the general case, i.e., loopy graphs, working with locally consistent beliefs that follow from the extremization of the first term does not guarantee that the factorized measure $\prod_{r \in R} b_{r}\left(\underline{x}_{r}\right)^{c_{r}}$ is properly normalized, therefore at the fixed point $\Delta F \neq 0$ generally. Nevertheless, in all the situations where it is meaningful to use message passing algorithms, we expect the corrections due to loops to be small, and for this very reason, also $\Delta F \ll F_{R}$.

As a consequence of the variational treatment, we now need to solve the set of equations

$$
\frac{\partial F_{R}[\{m\}]}{\partial m_{z}}=0 \quad \forall z .
$$

The precise form of the resulting equations, and what exactly they are enforcing depends on the choice made for the messages and how they appear in the belief equations. Next, we explain one possible choice that we retain as the natural one and we will call maximal message passing. 


\section{GAUGE-FREE 4M-CVM: MAXIMAL MESSAGES AND MOMENT MATCHING}

Previously [6,17-20], the set of messages have been defined using the so-called parent-to-child (P-t-C) approach. This means that messages $m_{\alpha \rightarrow \gamma}\left(\underline{x}_{\gamma}\right)$ are indexed by two regions labels, the father one $\alpha$ and the child one $\gamma$. We will say that a region $\alpha$ is father of $\gamma$, if $\alpha \supset \gamma$ and no region in $R$ is a subset of $\alpha$ and a superset of $\gamma$. In P-t-C no messages are considered from grandparents or higher ancestors.

While this approach is very systematic, it has the problem of introducing too many degrees of freedom in the test functions. As already mentioned, this may not have major consequences (aside from efficiency) in physical observables measured on a given instance, but introduces a gauge invariance that might be problematic in the comparison with the typical behavior of message passing equations in the average case scenario $[8,19]$. The reason is that in population dynamics one assumes messages arriving on a given region from different ancestors to be mostly uncorrelated: there are situations where this approximation is physically valid (e.g., when correlations are not too strong and regions are large enough), however, the gauge invariance implies messages can freely change under the gauge transformation and this introduces undesirable correlations among the messages. For this reason, a scheme free from the gauge invariance is very welcome.

We propose a top-down approach, that we call maximal message passing, in which messages to region $r$ flow from all its ancestors $p \supset r$. We prefer the maximal messages among other possibilities because they will allow us later to construct a gauge-free system of message passing equations.

Definition 1. Maximal messages are defined by the set of message functions used, and by how these functions participate in each region's belief, as follows:

(i) Every region $r \in R$ receives messages from all its ancestors $p \in A_{r}$. Messages are functions of the degrees of freedom in $r: m_{p \rightarrow r}\left(\underline{x}_{r}\right)$.

(ii) Message $m_{p \rightarrow \gamma}\left(\underline{x}_{\gamma}\right)$ will appear in the region partition function $z_{r}$ [i.e., Eq. (5)] of region $r$, iff $r \cap p=\gamma$.

The first point asserts that there are as many message functions as pairs of comparable regions in the CVM construction, and therefore the messages are labeled by these two regions as $m_{p \rightarrow r}\left(\underline{x}_{r}\right)$ where $r \subset p$. The term maximal messages comes since there are messages to every region from all its ancestors, not only the first direct parents as in parent to child. The second point defines $D_{r}$, the set of messages $m_{p \rightarrow \gamma}\left(\underline{x}_{\gamma}\right)$ that appear multiplicatively in the belief expression (or the partition function) of a certain region $r$, as

$$
D_{r}=\{p, \gamma \in R \mid p \nsubseteq r, p \cap r=\gamma \neq \varnothing\} .
$$

Put together, we have an expression for the beliefs at any region given by

$$
b\left(\underline{x}_{r}\right)=\frac{1}{z_{r}} e^{-\beta E_{r}\left(\underline{x}_{r}\right)} \prod_{p, \gamma \in D_{r}} m_{p \rightarrow \gamma}\left(\underline{x}_{\gamma}\right) .
$$

As a consequence also of the second point in the definition, any given message $m_{p \rightarrow r}$ is present in the belief equations of all regions whose intersection with $p$ is exactly $r$ :

$$
\partial m_{p \rightarrow r}=\left\{r^{\prime} \in R \mid r^{\prime} \cap p=r\right\} .
$$

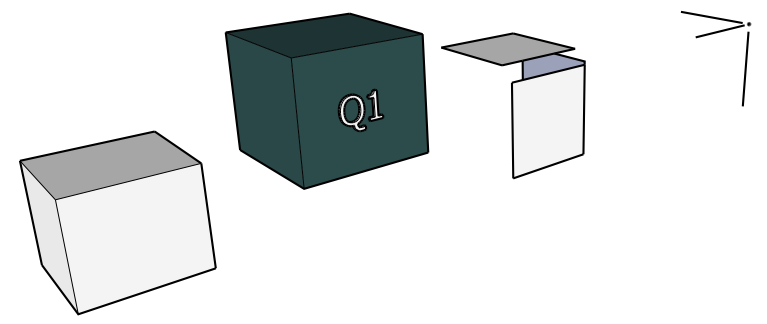

FIG. 2. Regions on whose beliefs the message $m_{Q_{1} \rightarrow s_{1}}\left(s_{1}\right)$ from the cubic region $Q_{1}$ to the central spin $s_{1}$ appear. On the leftmost diagram, $Q_{1}$ is still represented to help guiding the eye, but it does not belong to $\partial m_{Q_{1} \rightarrow s_{1}}$. The cube opposing $Q_{1}$, the three plaquettes and the three edges and $s_{1}$ itself, all intersect with $Q_{1}$ only at $s_{1}$, and therefore are in $\partial m_{Q_{1} \rightarrow s_{1}}$.

In order for this prescription to be valid, we have to show that it satisfies (4).

To keep with our previous 3D example, let us consider the case of the message $m_{Q_{1} \rightarrow s_{1}}\left(s_{1}\right)$, going from the cube $Q_{1}$ (dark) to the central spin $s_{1}$, as shown in Fig. 2. Then, the set of regions $\partial m_{Q_{1} \rightarrow s_{1}}$ on whose beliefs the message $m_{Q_{1} \rightarrow s_{1}}\left(s_{1}\right)$ appear are those whose intersection with $Q_{1}$ is exactly $s_{1}$, as represented in the diagram (Fig. 2).

\section{A. Properties of maximal messages}

Theorem 1. Equation (7) defines a valid GBP approximation on any set of regions $R$ defined by the cluster variational method.

This theorem is proved in Appendix A, based on the fact that the cluster variational method defines a partially ordered set and some properties relating the ancestors of a region and the set of equations in which messages to that region participate.

Extremal values of $F_{\mathrm{CVM}}$ are obtained by enforcing Eq. (6). Since all messages appear linearly, differentiating is equivalent to remove the messages from the equations in which they are present. In order to obtain a nicer presentation, we can solve instead

$$
m_{r_{0} \rightarrow \gamma}\left(\underline{x}_{\gamma}\right) \frac{\partial F_{R}[\{m\}]}{\partial m_{r_{0} \rightarrow \gamma}}=0
$$

which generates the following set of equations:

$$
\sum_{r \in \partial m_{r_{0} \rightarrow \gamma}} c_{r} \sum_{\underline{x}_{r} \backslash \underline{x}_{\gamma}} b_{r}\left(\underline{x}_{r}\right)=0 .
$$

Notice that, as a consequence of Eq. (4), a particular solution to this equation is found when each belief involved has the same marginal over the degrees of freedom $\underline{x}_{\gamma}$. Since beliefs are usually interpreted as approximations of the marginals of the joint probability distribution (1), we would require them to be consistent with one another. It is assuring to see that the consistency indeed is a solution of the extremal equations. In Appendix B, we prove the following.

Theorem 2. The extremal points of the approximated variational free energy $F_{R}[\{m\}]$ are found at consistent beliefs:

$$
\forall r \in R \quad \forall p \in A_{r} \quad b_{r}\left(\underline{x}_{r}\right)=\sum_{\underline{x}_{p} \backslash \underline{x}_{r}} b_{p}\left(\underline{x}_{p}\right) .
$$


From these equations we can write message passing update rules in different ways. Unfortunately, maximal message passing does not solve automatically the gauge invariance in the messages. Just as in P-t-C case, the introduced messages do not define the beliefs in a unique way, and many possible message values may represent the same beliefs. Aside from its nonoptimality as a representation, and probably derived efficiency and convergence problems, this invariance might be problematic for average case predictions, for instance, the prediction of critical temperatures in disordered systems.

Another relevant property of maximal message passing is that it is hierarchical.

Theorem 3. Let there be two CVM approximations for a given model. If one of the approximations is contained in the other, meaning that all regions in one are present in the other

$$
R_{\mathrm{CVM}_{1}} \subset R_{\mathrm{CVM}_{2}},
$$

then the beliefs in the smaller approximation (the less precise one) are obtained from the larger approximation by just setting to 1 all messages not common to both.

The proof is immediate since the definition of $D_{r}$ implies that $D_{r}^{1} \subset D_{r}^{2}$.

As a consequence, if one wants to recover the Bethe approximation from, e.g., the plaquette approximation, we only need to disregard (setting to 1) the plaquette-to-link and plaquette-to-spin messages in the belief and message passing equations. This property is also valid in the case of gauge-free maximal messages that we explain next. We recall, however, that two different approximations have different counting numbers, and therefore the free energy of the smallest approximation is not obtained by setting to zero the terms of the larger.

\section{B. Moment matching is gauge free}

The general way to create a message passing that is also gauge free starts from recognizing that the relevant quantities to match are not necessarily the belief as in Eq. (8) but their moments. Next, we present the case of Ising variables $s_{i}=$ \pm 1 . A more general presentation, regarding for instance Potts variables as in [22], is left for future work.

Let us go back to the messages. It has been shown in the context of P-t-C message passing $[18,19]$ that when the region graph contains loops, i.e., when from a bigger region there are two paths to get to a smaller region in the region graph, then the messages are not uniquely determined. In other words, since marginalization is transitive, forcing $b_{p} \rightarrow b_{r_{1}} \rightarrow b_{r_{0}}$ and $b_{p} \rightarrow b_{r_{2}} \rightarrow b_{r_{0}}$ is redundant. The marginalization $b_{r_{2}} \rightarrow$ $b_{r_{0}}$, for instance, automatically follows from the first chain of marginalizations and $b_{p} \rightarrow b_{r_{2}}$. In other words, interpreting the messages as Lagrange multipliers [6], the introduction of a multiplier to force $b_{r_{2}} \rightarrow b_{r_{0}}$ is unnecessary, and therefore, the set of multipliers is not uniquely determined.

A workaround to this problem has been given previously [17-19] where authors have identified a link between gauge invariance and loops in the region graph representation. At the end, it all amounts to discovering which are the redundant messages, and remove them from the representation, or set them to an arbitrary value, fixing the gauge $[17,18,20]$. In many cases, although the final objective is clear (destroying the loops), there are many different ways to achieve it, and each one has selected his own way. Next, we explain how to construct gauge-free message passing algorithms from scratch, not by destroying loops, but by restricting degrees of freedom in the messages. We specify a precise and unique way to do SO.

So far, maximal messages were introduced in full generality. Now, we will reduce their degrees of freedom as long as they keep ensuring the consistent marginalization of neighbor regions. Proceeding in this way, we do not affect the overall minimization of the CVM free energy.

We will now change perspective and interpret messages not as arbitrary functions enforcing beliefs consistency, but rather as a set of fields enforcing the agreement between the moments in the beliefs. For instance a message to a two-spin region can be rewritten as

$$
m_{p \rightarrow 1,2}\left(s_{1}, s_{2}\right)=e^{s_{1} s_{2} U_{p \rightarrow 1,2}+s_{1} u_{p \rightarrow 1}+s_{2} u_{p \rightarrow 2}} .
$$

The four values of function $m\left(s_{1}, s_{2}\right)$ are encoded into the three parameters $U, u_{1}, u_{2}$ since messages are insensitive to any normalization factor, a consequence of property in Eq. (4). Let us assume that the fields $u_{p \rightarrow 1}, u_{p \rightarrow 2}$ fix the correct first moments between the beliefs at region $p$ and at the two-spin region $(1,2)$, while $U$ determines the correlation. In such case, since all parents of region $(1,2)$ are sending messages to it, all those parents will have a first and second moment on variables $s_{1}$ and $s_{2}$ that are consistent to that of the belief at $(1,2)$ and therefore are consistent among them. This also means that given two ancestors of $(1,2)$, let us say $g, p \in A_{(1,2)}$ such that $g \in A_{p}$, the messages from $g$ to $p$ do not require fields of the type $u_{1}, u_{2}$, and $U_{1,2}$ any longer.

An example is handy. Take for instance the $2 \mathrm{D}$ square lattice, a small fraction of which is represented here

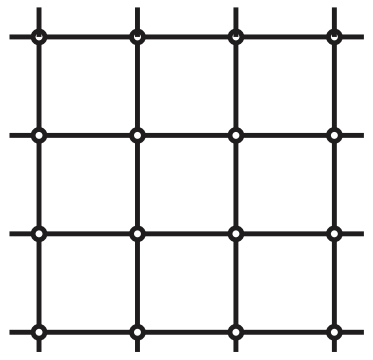

and the square plaquette-CVM approximation. Regions are the plaquettes, the links, and the spins (variables $s_{i}= \pm 1$ ) in the system. Any single spin receives messages of the form

$$
m_{r \rightarrow i}=e^{u_{r \rightarrow i} s_{i}}
$$

from the four links and the four plaquettes it belongs to. In the diagram, only the fields coming from one plaquette and two links are shown:

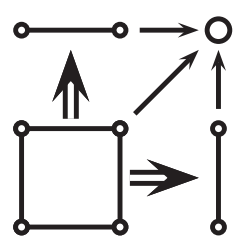

This ensures that the first moment of the beliefs in the plaquettes and the links are consistent with the first moment 
of the belief at spin $i,\left\langle s_{i}\right\rangle=\sum_{s_{i}} s_{i} b_{i}\left(s_{i}\right)$. Therefore, when plaquettes are sending messages to links (double arrows in diagram), they no longer need a multiplier (field) $u_{p \rightarrow i}$, and the message will only force the correlation

$$
m_{p \rightarrow(i j)}\left(s_{i}, s_{j}\right)=e^{U_{p \rightarrow i j} s_{i} s_{j}} .
$$

In such a way, even though the region graph has loops, the moments are not fixed redundantly, and the message passing is gauge free.

In general, a message $m_{p \rightarrow r}\left(\underline{s}_{r}\right)$ has $2^{|r|}-1$ degrees of freedom, where $|r|$ is the number of binary variables (spins) in region $r$. There are also $2^{|r|}-1$ non-null subsets of $r$, and therefore the same number of moments to describe a distribution over $|r|$ variables. The reduction of the degrees of freedom in the messages follows the following rule:

Definition 2. Moment matching. Message $m_{p \rightarrow r}\left(\underline{s}_{r}\right)$ contains a field $U_{q}$ enforcing the correlation among variables in $q \subseteq r$ as

$$
m_{p \rightarrow r}\left(\underline{s}_{r}\right)=e^{\cdots+U_{q}^{p \rightarrow r}} \prod_{i \in q} s_{i}+\cdots
$$

if and only if $r$ is the smallest region among all those containing the variables in $q$.

The smallest region containing $q$ is uniquely determined in the CVM construction thanks to the following properties: (i) the partial order defined by inclusion relations and (ii) the closure of the set of CVM regions under intersections of sets (the proof is easy and left to the reader).

Extreme values of the approximated free energy $F_{\mathrm{CVM}}$ can now be obtained by differentiating directly with respect to the fields $U_{q}$ that define the messages:

$$
\frac{\partial F_{\mathrm{CVM}}[\{U\}]}{\partial U_{q}^{p \rightarrow \gamma}}=0
$$

which generates the following set of equations:

$$
\sum_{r \in \partial m_{p \rightarrow \gamma}} c_{r}\left\langle\prod_{i \in q} s_{i}\right\rangle_{b_{r}}=0
$$

and

$$
\langle\ldots\rangle_{b_{r}}=\sum_{\underline{s}_{r}} \ldots b_{r}\left(\underline{s}_{r}\right) .
$$

Obviously, a particular solution is found when all distributions share the same moments over common degrees of freedom since Eq. (4) holds. In such case, all beliefs are also consistent with inner regions. It remains to show that this is in fact the only solution, which is the argument of the following:

Theorem 4. Maximal messages with moment matching fields ensures the consistency of beliefs.

The previous theorem states that the moment matching fields are enough to guarantee consistency. The next one completes our task by stating that indeed we need all of these fields to do so.

Theorem 5. Maximal messages with moment matching fields is gauge free.

Both theorems are proved in Appendix C.

\section{PLAQUETTE-CVM FOR ISING 2D}

Let us start by a simple case. The Ising ferromagnet, in the absence of external fields, is defined by the Hamiltonian

$$
\mathcal{H}(\underline{s})=-J \sum_{\langle i, j\rangle} s_{i} s_{j},
$$

where $\langle i, j\rangle$ defines nearby spins, and is given by the topology in which the system is embedded, and the degrees of freedom are $s_{i}= \pm 1$. The interaction constant $J$ is normally set to $J=1$.

Although the 2D case of this model has been exactly solved [26], we still can try our approximation on it, before moving to the unsolved higher dimensions. The first approximation beyond mean field and Bethe is the one containing all square plaquettes (the basic cell) as maximal regions. The cluster variation method then prescribes a free energy in terms of plaquette, link, and spin regions [19], with counting numbers $c_{P}=1, c_{L}=-1, c_{i}=1$, respectively.

The gauge-free $4 \mathrm{M}-\mathrm{CVM}$ is then written in terms of messages going from plaquettes to the links and spins interior to it. Beliefs are defined as follows:

$$
\begin{aligned}
& b_{P}\left(\underline{s}_{P}\right)= \frac{1}{z_{P}} e^{-\beta E_{4}\left(\underline{s}_{P}\right)} \prod_{L \in P} \prod_{\substack{P^{\prime} \supset L \\
P^{\prime} \neq P}} m_{P^{\prime} \rightarrow L}\left(s, s^{\prime}\right) \\
& \times \prod_{s \in P} \prod_{P^{\prime} \cap P=s} m_{P^{\prime} \rightarrow s}(s) \\
& \times \prod_{\substack{L \notin P \\
L \cap P \neq \varnothing}} m_{L \rightarrow s=L \cap P}(s), \\
& b_{L}\left(s, s^{\prime}\right)=\frac{1}{z_{L}} e^{-\beta E_{2}\left(s, s^{\prime}\right)} \prod_{\substack{P \supset L \\
m_{P \rightarrow L}}} m_{\left.P, s^{\prime}\right)} \\
& \times \prod_{\substack{\prod^{\prime} \in L \\
P^{\prime} \cap L=s}} m_{P^{\prime} \rightarrow s}(s) \\
& \prod_{\substack{L^{\prime} \neq L \\
L^{\prime} \cap L \neq \varnothing}} m_{L^{\prime} \rightarrow s=L^{\prime} \cap L}(s), \\
& b_{s}(s)=\frac{1}{z_{s}} e^{-\beta E_{1}(s)} \prod_{P \supset s} m_{P \rightarrow s}(s) \prod_{L \supset s} m_{L \rightarrow s}(s) .
\end{aligned}
$$

Graphically, the beliefs of each region are given by

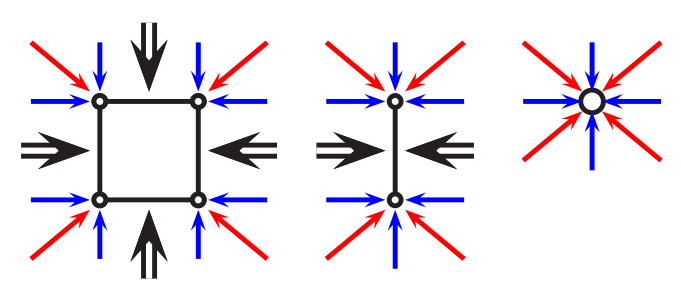

where double arrows represent messages to links $m_{P \rightarrow L}\left(s, s^{\prime}\right)$, oblique arrows (red in online version) messages from plaquettes to spins $m_{P \rightarrow s}(s)$ and remaining arrows (blue online) messages from links to spins $m_{L \rightarrow s}(s)$. 


\section{A. Message passing}

Message passing equations can be obtained in two different but equivalent ways:

(i) Old way: by imposing the consistency among beliefs, in this case some of the following:

$$
\begin{aligned}
b_{L}\left(s_{1}, s_{2}\right) & =\sum_{s_{3}, s_{4}} b_{P}\left(s_{1}, s_{2}, s_{3}, s_{4}\right), \\
b_{s}\left(s_{1}\right) & =\sum_{s_{2}, s_{3}, s_{4}} b_{P}\left(s_{1}, s_{2}, s_{3}, s_{4}\right), \\
b_{s}\left(s_{1}\right) & =\sum_{s_{2}} b_{L}\left(s_{1}, s_{2}\right) .
\end{aligned}
$$

(ii) New way: by imposing consistency among the moments of the distributions:

$$
\begin{aligned}
\sum_{s_{1}, s_{2}} s_{1} s_{2} b_{L}\left(s_{1}, s_{2}\right) & =\sum_{s_{1}, s_{2}, s_{3}, s_{4}} s_{1} s_{2} b_{P}\left(s_{1}, s_{2}, s_{3}, s_{4}\right), \\
\sum_{s_{1}} s_{1} b_{s}\left(s_{1}\right) & =\sum_{s_{1}, s_{2}, s_{3}, s_{4}} s_{1} b_{P}\left(s_{1}, s_{2}, s_{3}, s_{4}\right), \\
\sum_{s_{1}} s_{1} b\left(s_{1}\right) & =\sum_{s_{2}} s_{1} b_{L}\left(s_{1}, s_{2}\right) .
\end{aligned}
$$

Furthermore, as can be easily checked, not all three equations in the old way are independent: the third equation is consequence of the first two. This is the very reason why we reduced the amount of fields. In the new way there are only three values being fixed, and they are all independent. Both ways, however, produce the same update equations (message passing) independently of whether the messages have been reduced to be gauge fixed or are in full generality.

For instance, forcing any link belief $b_{L}\left(s_{1}, s_{2}\right)$ to marginalize onto one of its spins results in the following equation:

$$
\begin{aligned}
& \sum_{s_{2}} e^{\beta J s_{1} s_{2}} m_{P_{1} \rightarrow L}\left(s_{1}, s_{2}\right) m_{P_{2} \rightarrow L}\left(s_{1}, s_{2}\right) \\
& \quad \times m_{P_{3} \rightarrow s_{2}}\left(s_{2}\right) m_{P_{4} \rightarrow s_{2}}\left(s_{2}\right) \prod_{\substack{L^{\prime} \supset s_{2} \\
L^{\prime} \neq L}} m_{L^{\prime} \rightarrow s_{2}}\left(s_{2}\right) \\
& \propto m_{L \rightarrow s_{1}}\left(s_{1}\right) \prod_{P \supset L} m_{P \rightarrow s_{1}}\left(s_{1}\right),
\end{aligned}
$$

where we put a sign of proportionality $\propto$ instead of equality since messages are undefined by a multiplicative constant. These equations can be derived graphically using the representations of the beliefs and messages introduced above. The rules are quite simple. Interactions are represented by the rods, degrees of freedom by the circles, and messages by the arrows. If an interaction or a message appears in both sides of the equations, it can be canceled out. The degrees of freedom over which the marginalization is carried appear as full black circles. For instance, Eq. (13) is represented as in Fig. 3. The plaquette to link marginalization produces consistency relation between messages as shown in Fig. 4.

As can be seen, in either case (link to spin and plaquette to spin) the messages in the right hand side do not appear isolated. Consistency equations force the product of messages.

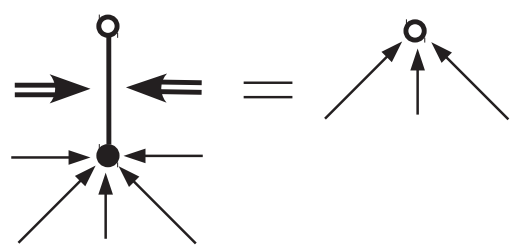

FIG. 3. Consistency equation between link beliefs and spin beliefs. Mathematically, it corresponds to the first two equations in (15) for $d=2$.

We could have used plaquette to spin marginalization as well, and the situation still would be similar. In such cases, it is left to the programmer to decide which iterative updating rule she wishes to implement to solve the consistency equations in a message passing way. She could, for instance, use the link to spin equation to update both plaquette to spin messages in a symmetric way, and then use the plaquette to spin equation to update the plaquette to link message. Let us emphasize that this freedom on the implementation of message passing equations remains even when the gauge is fixed, just as any fixed point equation can be written in infinite many ways. The gauge fixed property refers to the unicity of field values at a given fixed point, not to the strategies to find them.

If messages are considered in full generality, then we have a redundant description

$$
\begin{aligned}
m_{P \rightarrow L}\left(s_{1}, s_{2}\right) & =e^{U_{P \rightarrow L} s_{1} s_{2}+u_{P \rightarrow 1} s_{1}+u_{P \rightarrow 2} s_{2}}, \\
m_{L \rightarrow 1}\left(s_{1}\right) & =e^{u_{L \rightarrow 1} s_{1}}, \\
m_{L \rightarrow 2}\left(s_{2}\right) & =e^{u_{L \rightarrow 2} s_{2}}
\end{aligned}
$$

leading to a gauge invariance transformation involving $u$ messages [19]. On the contrary, using the gauge-free moment matching prescription previously defined in Definition 2, messages are

$$
\begin{aligned}
m_{P \rightarrow L}\left(s_{1}, s_{2}\right) & =e^{U_{P \rightarrow L} s_{1} s_{2}}, \\
m_{P \rightarrow 1}\left(s_{1}\right) & =e^{u_{P \rightarrow 1} s_{1}}, \\
m_{L \rightarrow 1}\left(s_{1}\right) & =e^{u_{L \rightarrow 1} s_{1}} .
\end{aligned}
$$

Details of the update equations and an example on 2D single instance are given next.

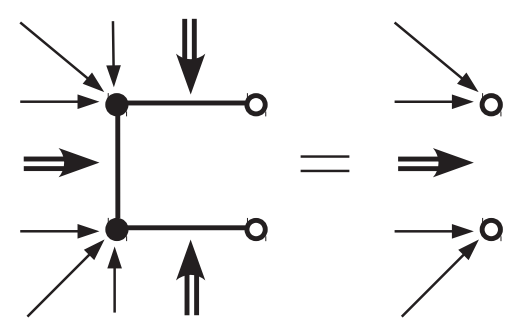

FIG. 4. Consistency equation between plaquette beliefs and link beliefs. Mathematically, it corresponds to the first two equations in (15) for $d=2$. 


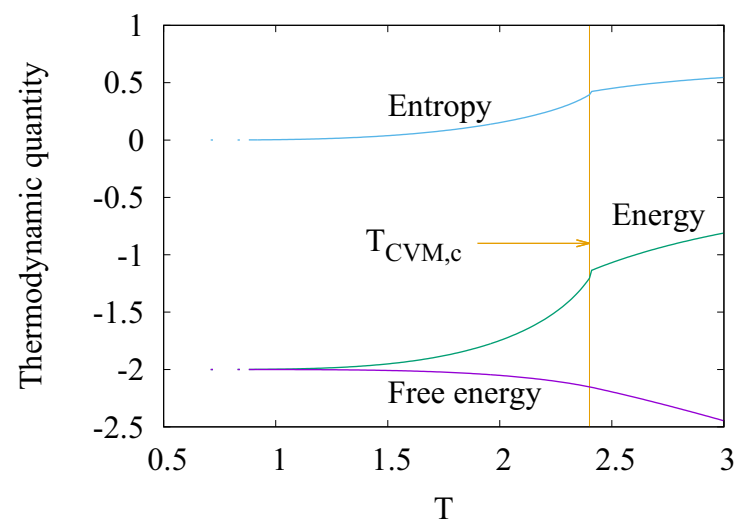

FIG. 5. Estimated intensive thermodynamic quantities for the Ising 2D model using gauge-free message passing on the plaquetteCVM approximation. The vertical line marks the plaquette-CVM approximate critical temperature for this model.

\section{B. 2D single instance implementation}

The self-consistent message passing equations can be written as $u_{L}=\hat{u}_{L}\left(\beta, J, u_{L}, U, u_{P}\right)$ and $U=\hat{U}\left(\beta, J, u_{L}, U, u_{P}\right)$, $u_{P}=\hat{u}_{P}\left(\beta, J, u_{L}, U, u_{P}\right)$, where

$$
\begin{aligned}
\hat{u}_{L} & =\frac{1}{2} \ln [K(1) / K(-1)]-u_{P_{a}}-u_{P_{b}}, \\
\hat{u}_{P} & =\frac{1}{4} \ln \left[\frac{K(1,1) K(1,-1)}{K(-1,1) K(-1,-1)}\right]-u_{L}, \\
\hat{U} & =\frac{1}{4} \ln \left[\frac{K(1,1) K(-1,-1)}{K(1,-1) K(-1,1)}\right] .
\end{aligned}
$$

The $K(\ldots)$ terms are partial traces over the spins in the plaquette and link, given by

$$
\begin{aligned}
K\left(s_{1}\right)= & \sum_{s_{2}} e^{\left(\beta J_{12}+U_{1}+U_{2}\right) s_{1} s_{2}+\left(u_{P_{1}}+u_{P_{2}}+u_{L 1}+u_{L 2}+u_{L 3}\right) s_{2}} \\
K\left(s_{1}, s_{2}\right)= & \sum_{s_{3}, s_{4}} \exp \left[s_{2} s_{3}\left(\beta J_{23}+U_{23}\right)+s_{1} s_{4}\left(\beta J_{14}+U_{14}\right)\right. \\
& +s_{3} s_{4}\left(\beta J_{34}+U_{34}\right) \\
& +\left(u_{P_{4}}+u_{L_{1} \rightarrow 4}+u_{L_{2} \rightarrow 4}\right) s_{4} \\
& \left.+\left(u_{P_{3}}+u_{L_{1} \rightarrow 3}+u_{L_{2} \rightarrow 3}\right) s_{3}\right]
\end{aligned}
$$

in correspondence with the fields in the left hand sides of Figs. 3 and 4.

The implementation of the message passing is carried by randomly selecting a plaquette (or link) and updating their fields as prescribed by Eq. (14). In 2D Ising model we obtain the expected results (see Fig. 5). Above the approximation critical temperature (not exact) $T_{c}=1 / \beta_{c} \simeq 2.43$ all fields acting on single spins are zero $u_{L \rightarrow i}=u_{P \rightarrow i}=0$, and the system is in a paramagnetic phase with zero global magnetization. In this range, the only nonzero field is the correlation field $U_{P \rightarrow L}$ (a detailed studied of this phase is in [27]). Below $T_{c}$, the system is in a ferromagnetic phase, with nonzero fields over spins and local as well as global magnetization.
Next, we show how to generalize this method to compute the critical temperature of the Ising model in general dimension, under the plaquette-CVM approximation.

\section{Critical temperature for Ising $\boldsymbol{d}$ dimensional}

Let us focus on the case of the plaquette-CVM approximation in the general $d$-dimensional Ising model on the hypercubic lattice. This case includes the model of the previous section.

We will show how to obtain analytic expression for the critical temperature of the ferromagnetic model in this approximation at all dimensions and, furthermore, we will show that the asymptotic behavior is correctly until the third order in $1 / d$, therefore being equivalent to the loop corrections of [23].

The plaquette approximation is the one that uses plaquettes as the biggest regions. In such case, the counting numbers of the plaquettes are always $c_{P}=1$. Every link belongs to $2(d-1)$ plaquettes and, therefore, its counting number is $c_{L}=1-2(d-1)$. Every spin belongs to $2 d$ links and $2 d(d-1)$ plaquettes and have counting number $c_{s}=$ $1-2 d(d-1)-2 d[1-2(d-1)]=1-2 d(2-d)$. Beliefs, therefore, have the following schematic representation, where, as usual, double arrows are messages from plaquette to link, oblique arrows from plaquette to spin, and remaining (vertical and horizontal) arrows are from links to spins.

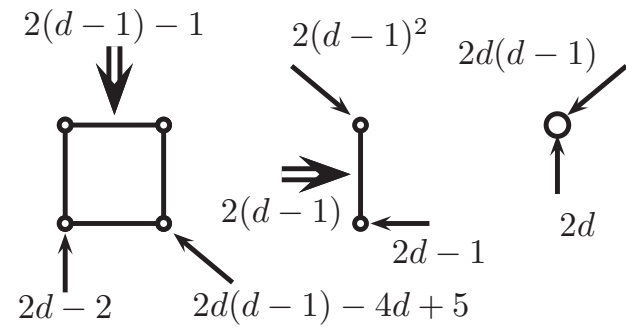

For clarity, only one type of message of each type is represented in each region together with the number of such messages that enter in the belief equation of that region. However, the reader should keep in mind that, for instance, there are $2 d(d-1)-4 d+5$ plaquette-to-spin fields entering at very corner of the represented plaquette.

In general, consistency equations for messages keep the same structure represented graphically in the previous section, but only the amount of messages entering every region changes. Exploiting the isotropy of the model, we can look for fixed points in which all messages are the same. In other words, we will assume that all link to spin messages are characterized by a unique field $u_{L}$, while plaquette to link messages by the field $U$ and plaquette to spin messages by $u_{P}$.

Graphically, the updating equations for the messages have the following representation:

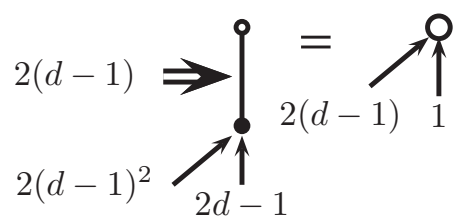




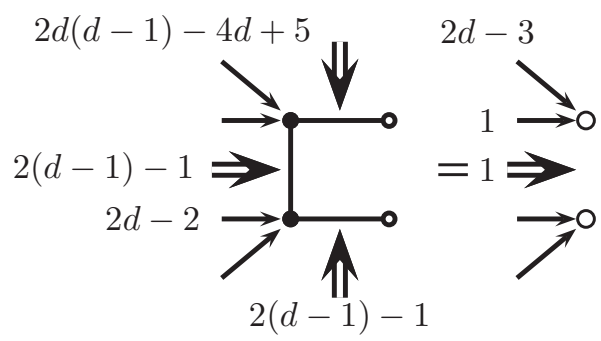

Exploiting the symmetry of the problem, we gain some clarity by showing only the multiplicity of one representative of each type of field. In the second diagram, fields not having a number by its side have the same multiplicity that is represented for its equivalent by a reflection along the horizontal axis.

Let us define

$$
\begin{aligned}
K\left(s_{1}\right)= & \sum_{s_{2}} e^{\{\beta J+[2(d-1) U]\} s_{1} s_{2}+\left[2(d-1)^{2} u_{P}+(2 d-1) u_{L}\right] s_{2}}, \\
K\left(s_{1}, s_{2}\right)= & \sum_{s_{3}, s_{4}} \exp \left(s_{2} s_{3}[\beta J+(2 d-3) U]\right. \\
& +s_{1} s_{4}[\beta J+(2 d-3) U]+s_{3} s_{4}[\beta J+(2 d-3) U] \\
& +\left\{[2 d(d-1)-4 d+5] u_{P}+(2 d-2) u_{L}\right\} s_{4} \\
& \left.+\left\{[2 d(d-1)-4 d+5] u_{P}+(2 d-2) u_{L}\right\} s_{3}\right) .
\end{aligned}
$$

In terms of this, the self-consistent equations can be written as

$$
\begin{aligned}
U & =\hat{U}\left(\beta, J, u_{L}, U, u_{P}\right) \\
& =\frac{1}{4} \ln \left[\frac{K(1,1) K(-1,-1)}{K(1,-1) K(-1,1)}\right] \\
u_{P} & =\hat{u}_{P}\left(\beta, J, u_{L}, U, u_{P}\right) \\
& =\frac{1}{2 d-3}\left\{\frac{1}{4} \ln \left[\frac{K(1,1) K(1,-1)}{K(-1,1) K(-1,-1)}\right]-u_{L}\right\}, \\
u_{L} & =\hat{u}_{L}\left(\beta, J, u_{L}, U, u_{P}\right) \\
& =\frac{1}{2} \ln [K(1) / K(-1)]-2(d-1) u_{P} .
\end{aligned}
$$

The solution to this set of equations is to be found numerically in general. A simpler case is that of the high temperatures, in which we suppose a paramagnetic phase characterized by $u_{L}=u_{P}=0$ and $U \neq 0$. In such case, the equation $U=\hat{U}$ becomes the simpler

$$
U=\operatorname{arctanh}\left(\{\tanh [(2 d-3) U+\beta J]\}^{3}\right) .
$$

This corresponds to the case treated in [27].

Moreover, the paramagnetic solution is the starting point to obtain the critical temperature of the system as the instability of the paramagnetic solution. Taking

$$
\mathbb{K}(\beta)=\left(\begin{array}{cc}
1-\frac{\partial \hat{u}_{L}}{\partial u_{L}} & \frac{\partial \hat{u}_{L}}{\partial u_{P}} \\
\frac{\partial \hat{u}_{P}}{\partial u_{L}} & 1-\frac{\partial \hat{u}_{P}}{\partial u_{P}}
\end{array}\right)_{u_{L}=0, U=\hat{U}, u_{P}=0}
$$

a continuous instability appears at the point in which $\mathbb{K}(\beta)$ is singular and therefore the critical temperature is defined as

$$
\operatorname{det} \mathbb{K}\left(\beta_{c}\right)=0 \text {. }
$$

Note that this is not fully analytical at this point since the numerical solution of (16) is still needed. However, after
TABLE I. Inverse critical temperatures of the Ising model on a regular hypercubic lattice in $d$ dimensions. In the second column, we report the best estimate for the true $\beta_{c}$, while the other columns contain the inverse critical temperatures in three different mean field approximations: the plaquette CVM discussed in this work, the loop corrected Bethe of Ref. [23], that can be computed only for $d>2$, and the standard Bethe approximation.

\begin{tabular}{lcccc}
\hline \hline & & Plaquette & Loop corrected & \\
CVM & True $\beta_{c}$ & CVMe & Bethe \\
\hline 2 & 0.440687 (exact) & 0.412258 & & 0.346574 \\
3 & $0.221654(6)[28]$ & 0.216932 & 0.238520 & 0.202733 \\
4 & $0.14966(3)[29]$ & 0.148033 & 0.151650 & 0.143841 \\
5 & $0.11388(3)[30]$ & 0.113362 & 0.114356 & 0.111572 \\
6 & & 0.092088 & 0.092446 & 0.091161 \\
\hline \hline
\end{tabular}

some transformations we obtain an analytic expression for the critical temperature at all dimensions $d>2$ :

$$
\beta_{\mathrm{CVM}}=\frac{1}{2} \ln \left[\left(\frac{d-2}{d}\right)^{d-2}\left(\frac{2 d-1}{2 d-3}\right)^{2 d-3}\right] .
$$

In the $d=2$ case, the solution is also analytical but given by

$$
\beta_{\mathrm{CVM}}(d=2)=\frac{1}{2} \ln \left(\frac{5+\sqrt{17}}{4}\right) \text {. }
$$

This prediction can be compared with known results. In Table I we show the best estimate of the true $\beta_{c}$ on a regular hypercubic lattice with $2 \leqslant d \leqslant 6$, together with the estimate from plaquette CVM, that from the Bethe approximation, where $\beta_{\text {Bethe }}=\operatorname{arctanh}\left[(2 d-1)^{-1}\right]$, and the one from Bethe with loop corrections due to Rizzo and Montanari [23]. In the latter approximation, the critical temperature can be computed only if $d>2$.

In the large $d$ limit, the plaquette-CVM critical temperature is correct up to the second order in the $1 / d$ expansion, exactly as the loop corrected Bethe approximation [23]

$$
\frac{1}{2 d \beta_{\mathrm{CVM}}}=\underbrace{\overbrace{1-\frac{1}{2 d}}^{\text {Bethe }}-\frac{1}{3 d^{2}}}_{\text {Loop corr. Bethe }}-\frac{5}{12 d^{3}}+\cdots
$$

while the standard Bethe approximation is correct only up to the $O(1 / d)$ term.

We find this result very interesting because it improves over the Bethe approximation by one order of magnitude in the $1 / d$ expansion, while still providing a very accurate critical temperature at $d=2$. On the contrary, the loop corrected Bethe approximation is divergent in $d=2$, and this makes the present 4M-CVM much more useful for the study of low dimensional systems.

\section{CONCLUSIONS}

We have shown how to create gauge-free message passing implementations of the cluster variational approximations for general models of spinlike variables. To do so, we presented a way of introducing the messages in the CVM that differs from standard parent-to-child messages in that messages are 
sent to a region from all its ancestors, and not only by its direct parents. While previous attempts to fix the gauge invariance in GBP equations $[17,18,20]$ relied on the idea of removing some selected messages from the equations, our approach increases the number of such messages, but with a restriction on their degrees of freedom.

This systematic restriction of message degrees of freedom automatically produces gauge-free variational approximations, such that there is a one-to-one correspondence between free energy minima and the values of the fields that define the messages. Furthermore, we put emphasis in an interpretation of the fields involved in the message passing as imposing consistency between moments of the local distributions (beliefs) rather than the usual interpretation of messages forcing consistent belief marginalization. We called the resulting method maximal messages with moment matching (4M-CVM).

The approach includes the Bethe approximation as the starting point, and improves it when larger regions are taken into consideration. We showed that the method produces sensible analytical results for the plaquette approximation of the critical temperature of the Ising ferromagnet in general dimensions, that correctly accounts for the next leading order in the high dimensional expansions, just as the more complicated loop calculus does [23].

\section{ACKNOWLEDGMENTS}

The authors want to thank the hospitality of E. Aurell and his group at Nordita, during the last part of the elaboration of this paper. We also thank D. de la Regata for support with 3D graphics. This work has been supported in part by the ERC under the European Unions 7th Framework Programme Grant Agreement No. 307087-SPARCS.

\section{APPENDIX A: MAXIMAL GBP IS ALWAYS VALID}

We will prove that independently of the regions chosen as maximal, the introduction of multiplicative messages from maximal regions to their children, as explained in the main text, generates a valid GBP. Valid means that Eq. (4) is satisfied. First of all, let us prove that (7) satisfies Eq. (4). Without loss of generality, let us focus on a given region $r_{0} \in R$ and one of its children regions $\alpha \subset r_{0}$. The message $m_{r_{0} \rightarrow \alpha}\left(\underline{x}_{\gamma}\right)$ appears in the belief equations of all regions $r$ such that $r_{0} \cap r=\alpha$, which defines

$$
B_{r_{0}, \alpha} \equiv \partial m_{r_{0} \rightarrow \alpha}=\left\{r^{\prime} \in R \mid r^{\prime} \cap r=\alpha\right\} .
$$

An example of $B_{r_{0}, \alpha}$ are the regions (except $Q_{1}$ ) appearing in the diagrams of Fig. 2.

The property we need to prove is [restating Eq. (4)] as follows:

Proposition 1.

$$
\forall_{r_{0} \in R_{0}} \forall \begin{gathered}
\alpha \in R \\
\alpha \subset r_{0}
\end{gathered} \quad \sum_{r^{\prime} \in B_{r_{0}, \alpha}} c_{r^{\prime}}=0 .
$$

This property is similar to the one defining the counting numbers (3), but not the same. We will show the validity of (A1) from that of Eq. (3). Let us start by restating Eq. (3) as

$$
\sum_{r \in A_{\alpha}^{o}} c_{r}=1
$$

where we have defined $A_{\alpha}^{o}$ the extended set of ancestors of any region $\alpha$ to include $\alpha$ itself:

$$
A_{\alpha}^{o} \equiv A_{\alpha} \cup\{\alpha\}
$$

The set $B_{r_{0}, \alpha} \subset A_{\alpha}^{o}$. Furthermore, we can write

$$
A_{\alpha}^{o}=B_{r_{0}, \alpha}+\bar{B}_{r_{0}, \alpha}^{o},
$$

where the sets in the right hand side are disjoint, and as follows:

Definition 3. $\bar{B}_{r_{0}, \alpha}^{o}$ :

$$
\bar{B}_{r_{0}, \alpha}^{o}=\left\{r \in R \mid r \cap r_{0}>\alpha\right\}
$$

is the set of all ancestors of $\alpha$ such that their intersection with $r_{0}$ is larger than $\alpha$.

The part $\bar{B}_{r_{0}, \alpha}^{o}$ contains the ancestors of $\alpha$ in whose beliefs the message $m_{r_{0} \rightarrow \alpha}$ does not appear, and it will never be an empty set since it includes at least $r_{0}$ and its ancestry. In Fig. 2, $\bar{B}_{r_{0}, \alpha}^{o}$ is exactly the absent part with respect to Fig. 1, including also $Q_{1}$.

From now on we will use relational operators $>,<, \geqslant$, $\leqslant$ freely since the hierarchy established by the inclusion of sets in the regions defines a partially ordered set. In this sense, $\alpha<\beta$ means that $\alpha \subset \beta$ and $\alpha \neq \beta$.

Since the sets $B_{r_{0}, \alpha}, \bar{B}_{r_{0}, \alpha}^{o}$ are disjoint, the sum (A2) can be split among them, becoming

$$
\sum_{r \in B_{r_{0}, \alpha}} c_{r}+\sum_{r \in \bar{B}_{r_{0}, \alpha}^{o}} c_{r}=1 .
$$

If $\bar{B}_{r_{0}, \alpha}^{o}=A_{r_{0}}^{o}$, then Proposition 1 is proved since the second sum will equal 1 . Otherwise, the validity of the maximal messages [Eq. (A1)] falls from proving that in the most general case

$$
\sum_{r \in \bar{B}_{r_{0}, \alpha}^{o}} c_{r}=1,
$$

as we will see through the rest of the appendix.

Let us see some properties of the set $\bar{B}_{r_{0}, \alpha}^{o}$. From now on, all greek letters $\alpha, \beta, \gamma \ldots$ refer to regions in $\bar{B}_{r_{0}, \alpha}^{o}$.

Lemma 1. $\bar{B}_{r_{0}, \alpha}^{o}$ is a finite partially ordered set.

Proof. Since $\bar{B}_{r_{0}, \alpha}^{o} \subset R$, it is finite. Furthermore, the set of all regions $R$ itself is a partially ordered set, defined by the inclusion relation $r_{1}<r_{2} \Longleftrightarrow r_{1} \subset r_{2} \wedge r_{1} \neq r_{2}$.

From now on we will use the terminology of partially ordered sets. For instance, we will say that region $r_{2}$ covers $r_{1}$ if $r_{2}>r_{1}$ and there is no $z$ such that $r_{2}>z>r_{1}$.

Lemma 2. $\bar{B}_{r_{0}, \alpha}^{o}$ is closed under intersection with $r_{0}$.

Proof. The set of regions generated by the cluster variational method is closed under intersections in general. Let $\gamma \in \bar{B}_{r_{0}, \alpha}^{o}$, then $\gamma>\alpha$ and $\gamma \cap r_{0}=\eta>\alpha$. Then, trivially, $\eta \cap r_{0}=\eta>$ $\alpha$ which guarantees that $\eta \in \bar{B}_{r_{0}, \alpha}^{o}$

Since $\eta<r_{0}$, note the following corollaries.

Corollary 1. All $\beta_{i} \in \bar{B}_{r_{0}, \alpha}^{o}$ such that $\beta_{i}$ covers $\alpha$ inside $\bar{B}_{r_{0}, \alpha}^{o}$, are subsets of $r_{0}\left(\beta_{i}<r_{0}\right)$.

Corollary 2.

$$
B=\left\{\beta_{i} \in \bar{B}_{r_{0}, \alpha}^{o} \mid \beta_{i} \text { covers } \alpha \text { in } \bar{B}_{r_{0}, \alpha}^{o}\right\}
$$

is the set of minimal elements in $\bar{B}_{r_{0}, \alpha}^{o}$. 
Lemma 3. If $\gamma \in \bar{B}_{r_{0}, \alpha}^{o}$ then $A_{\gamma}^{o} \subset \bar{B}_{r_{0}, \alpha}^{o}$.

Proof.

$$
\forall_{\eta \in A_{\gamma}^{o}} \eta \cap r_{0} \geqslant \gamma>\alpha \Rightarrow \eta \in \bar{B}_{r_{0}, \alpha}^{o}
$$

As a consequence, the entire $\bar{B}_{r_{0}, \alpha}^{o}$ is generated by the ancestry of members of $B$, this is as follows:

Lemma 4.

$$
\bar{B}_{r_{0}, \alpha}^{o}=\bigcup_{\beta_{i} \in B} A_{\beta_{i}}^{o} .
$$

We have written $\bar{B}_{r_{0}, \alpha}^{o}$ in terms of the set of ancestors of some minimal elements $\beta_{1}, \beta_{2} \ldots$ We emphasize that all such ancestries share, at least, the common element $r_{0}$ and its ancestry. Therefore, they are not disjoint sets.

In order to prove (A4) we start from the fact that

$$
\forall_{\beta \in R} \sum_{r \in A_{\beta}^{o}} c_{r}=1 .
$$

Furthermore, every time that the intersection of ancestries is not empty, which is the case of all minimal elements $\beta_{1}, \beta_{2} \ldots$ since they all share $r_{0}$, there exists an element in $\gamma \in \bar{B}_{r_{0}, \alpha}^{o}$, such that $A_{\gamma}^{o}=A_{\beta_{i}}^{o} \cap A_{\beta_{j}}^{o}$. Let us formalize and generalize this idea.

Let us use the definition of least upper bound $\phi=\operatorname{lub}(\gamma, \eta)$ as the smallest element in $\bar{B}_{r_{0}, \alpha}^{o}$ that is both $\phi \geqslant \gamma$ and $\phi \geqslant \eta$. By minimum we mean that every other $z$ that shares both properties happens to be $z>\eta$. In finite posets, the least upper bound might not exist, but if it does, it is uniquely defined.

We will show that the intersections of the ancestries of elements in $\bar{B}_{r_{0}, \alpha}^{o}$ can be written themselves as the ancestry of another element in $\bar{B}_{r_{0}, \alpha}^{o}$.

Lemma 5 (The intersection of ancestries). Let $\gamma_{1}, \gamma_{2} \in \bar{B}_{r_{0}, \alpha}^{o}$ with a non-null intersection of their ancestors $A_{\gamma_{1}}^{o} \cap A_{\gamma_{2}}^{o} \neq \varnothing$, then,

$$
\begin{aligned}
\exists \eta & =\operatorname{lub}\left(\gamma_{1}, \gamma_{2}\right) \in \bar{B}_{r_{0}, \alpha}^{o} \\
\text { such that } \quad A_{\eta}^{o} & =A_{\gamma_{1}}^{o} \cap A_{\gamma_{2}}^{o} .
\end{aligned}
$$

Proof. If the intersection of ancestries is not null, then it has at least one element, let us say $\theta$. Since $\gamma_{1}, \gamma_{2} \leqslant \theta$, then all ancestors of $\theta$ are also comparable and above $\gamma_{1}$ and $\gamma_{2}$. In other words, $\theta \in A_{\gamma_{1}}^{o} \cap A_{\gamma_{2}}^{o} \Rightarrow A_{\theta}^{o} \subset A_{\gamma_{1}}^{o} \cap A_{\gamma_{2}}^{o}$. Let us suppose that $A_{\gamma_{1}}^{o} \cap A_{\gamma_{2}}^{o}$ is the union of more than one ancestries of many incomparable $\theta_{i}$ 's. This cannot be the case, since the intersection of any two $\theta_{1}$ and $\theta_{2}$ produces a lower $\theta$, that is again in $A_{\gamma_{1}}^{o} \cap A_{\gamma_{2}}^{o}$ and whose ancestry includes that of both $\theta_{1}$ and $\theta_{2}$. Repeating this procedure, we end up with a unique value $\eta$, such that $A_{\eta}^{o}=A_{\gamma_{1}}^{o} \cap A_{\gamma_{2}}^{o}$. The fact that $\eta=\operatorname{lub}\left(\gamma_{1}, \gamma_{2}\right)$ is trivial.

Since the set $\bar{B}_{r_{0}, \alpha}^{o}$ is written as the union of ancestries of the minimal sets $\beta_{1}, \beta_{2}, \ldots$ (see Lemma 4 ), then from the previous lemma and the fact that all ancestries of the minimal elements share at least $r_{0}$ and its ancestry, note the following:

Corollary 3. Let $\Pi$ be any subset of the indices of the minimal sets $\beta_{1}, \beta_{2}, \ldots$ covering $\alpha$ inside $\bar{B}_{r_{0}, \alpha}^{o}$, then

$$
\exists_{r \in \bar{B}_{r_{0}, \alpha}^{o}} \bigcap_{i \in \Pi} A_{\beta_{i}}^{o}=A_{r}^{o} .
$$

Now, using the inclusion exclusion principle, we can write

$$
\begin{aligned}
\sum_{r \in \bar{B}_{r_{0}, \alpha}^{o}} c_{r}= & \sum_{\beta \in B} \sum_{\gamma \in A_{\beta}^{o}} c_{\gamma}-\sum_{\beta_{1}, \beta_{2} \in B} \sum_{\gamma \in A_{\beta_{1}}^{o} \cap A_{\beta_{2}}^{o}} c_{\gamma} \\
& +\sum_{\beta_{1}, \beta_{2}, \beta_{3} \in B} \sum_{\gamma \in A_{\beta_{1}}^{o} \cap A_{\beta_{2}}^{o} \cap A_{\beta_{3}}^{o}} c_{\gamma}-\ldots,
\end{aligned}
$$

where every second sumatory is itself of the form

$$
\sum_{r \in A_{\gamma}^{o}} c_{r}=1
$$

by the previous corollary. So, if the set $\bar{B}_{r_{0}, \alpha}^{o}$ is generated by the ancestries of $K$ minimal elements $\beta_{i}$, then

$$
\begin{aligned}
\sum_{r \in \bar{B}_{r_{0}, \alpha}} c_{r} & =\left(\begin{array}{c}
K \\
1
\end{array}\right)-\left(\begin{array}{l}
K \\
2
\end{array}\right)+\left(\begin{array}{l}
K \\
3
\end{array}\right)-\ldots+(-1)^{K+1}\left(\begin{array}{l}
K \\
K
\end{array}\right) \\
& =1+(1-1)^{K}=1,
\end{aligned}
$$

which concludes the proof of (A4) and from it that of (A1)

To help visualize a little bit the rather complicated algebra of sets, we depict in Fig. 6 the Hasse diagram and the sets $\bar{B}_{r_{0}, \alpha}^{o}$ and $B_{r_{0}, \alpha}$ for the cube-CVM approximation for the 3D square lattice spin model. In particular, the case of the message going from the cubic region $Q_{1}$ to the central spin region $s_{1}$ is shown.

\section{APPENDIX B: MARGINALIZATION OF BELIEFS}

Extremization of the variational free energy with respect to the message functions results in the following equation:

$$
\sum_{r \in \partial m_{r_{0} \rightarrow \gamma}} c_{r} \sum_{\underline{x}_{r} \backslash \underline{x}_{\gamma}} b_{r}\left(\underline{x}_{r}\right)=0
$$

for each message $m_{r_{0} \rightarrow \gamma}$.

In this appendix, we show that the only solution for such set of equations is beliefs satisfying

$$
\forall_{p \in A_{r}} b_{r}\left(\underline{x}_{r}\right)=\sum_{\underline{x}_{p} \backslash \underline{x}_{r}} b_{p}\left(\underline{x}_{p}\right) .
$$

We will prove this in an inductive manner, assuming that starting from the maximal regions onto a certain level, all regions marginalize and from this assumption, we will show that the next level also correctly marginalizes.

Induction: Base case. Let $r_{0}$ be a region whose ancestry $A_{r_{0}}$ consists only on maximal regions. Then, the intersection of members of any two members of $A_{r_{0}}$ cannot be smaller than $r_{0}$ since by definition $r \in A_{r_{0}} \Rightarrow r_{0} \subset r$, but the intersection cannot be larger than $r_{0}$ since in such case $\gamma=r_{1} \cap r_{2}>r_{0}$ would be an ancestor of $r_{0}$ that is not a maximal region. So, any two elements of $A_{r_{0}}$ intersect exactly at $r_{0}$.

Given the definition of the set of regions in whose belief a given message is present

$$
\partial m_{p \rightarrow r}=\left\{r^{\prime} \mid r^{\prime} \cap p=r\right\},
$$

the previous result implies that any message $m_{r_{1} \rightarrow r_{0}}\left(\underline{x}_{r 0}\right)$ from a parent $r_{1}$ of $r_{0}$ appears in the belief of all the other parents 


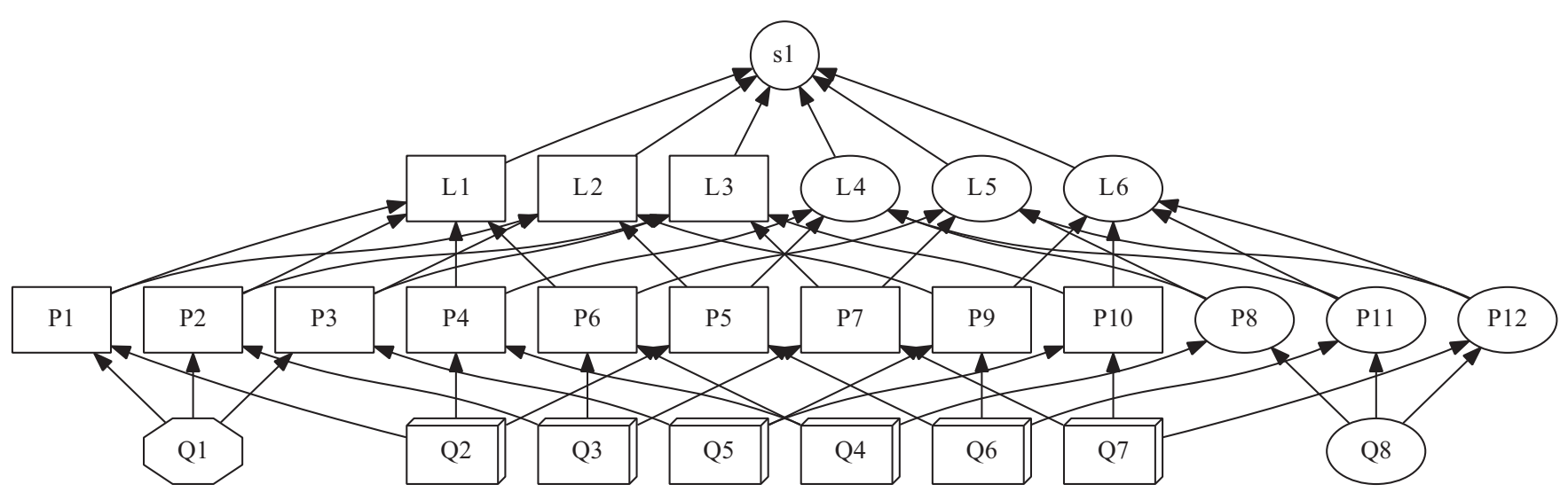

FIG. 6. Hasse diagram of the regions in the 3D cube approximation, for the 3D Ising model. Variables live in the nodes of a tridimensional cubic lattice. Maximal regions are the cubes (basic cell), and from them all intersections generate new regions, as prescribed by cluster variational method (see also Fig. 1). In this representation, all regions containing one central spin $s_{1}$ are depicted. Central spin $s_{1}=\alpha$ is surrounded by eight maximal cubic regions $Q_{1}, \ldots, Q_{8}$. We represent the partially ordered set defined by the inclusion relations among the ancestors of $s_{1}$. Arrows point in the parent-to-child direction. Bottom figures are the eight cubes, next level are all the square plaquettes that intersect among these cubes, the third layer is made of the six links containing spin $s_{1}$. We consider $Q_{1}=r_{0}$ to be the region sending message to spin $s_{1}$. Shapes correspond to the position in the ancestry of $s_{1}$ with respect to the message $m_{Q_{1} \rightarrow s_{1}}$. All circular regions represent elements of $B_{r_{0}, \alpha}$, and therefore their intersection with $Q_{1}$ is exactly $s_{1}$ (represented also in Fig. 2, except for $Q_{1}$ ). Angular regions are those in $\bar{B}_{r_{0}, \alpha}^{o}$ (the absent part of Fig. 2, including $Q_{1}$ ).

except $r_{1}$ itself and including $r_{0}$. Mathematically,

$$
\forall_{r \in A_{r_{0}}} \partial m_{r \rightarrow r_{0}}=\left\{r_{0}\right\} \cup A_{r_{0}} \backslash\{r\}=A_{r_{0}}^{o} \backslash\{r\} .
$$

Let there be $\left|A_{r_{0}}\right|=K$ parents to $r_{0}$. We have $K$ equations of the type (B1):

$$
\forall_{i \in[1,2, \ldots, K]} \sum_{r \in A_{r_{0}}^{o} \backslash\left\{r_{i}\right\}} c_{r} \sum_{\underline{x}_{r} \backslash \underline{x}_{0}} b_{r}\left(\underline{x}_{r}\right)=0 .
$$

Except for $c_{r_{0}}=1-K$, all other counting numbers are $c_{r}=1$. Furthermore, we can add the missing summand in each case, to obtain, for each $i \in[1,2, \ldots, K]$,

$$
(1-K) b_{0}\left(\underline{x}_{0}\right)+\sum_{r \in A_{r_{0}}} \sum_{\underline{x}_{r} \backslash \underline{x}_{0}} b_{r}\left(\underline{x}_{r}\right)=\sum_{\underline{x}_{i} \backslash \underline{x}_{0}} b_{r_{i}}\left(\underline{x}_{i}\right) .
$$

Now, the left hand side is independent of $i$, and therefore all right hand sides have to be equal for every $i$. This proves that the parents are consistent among each other on their belief at region $r_{0}$. To show that they agree with $b_{0}\left(\underline{x}_{0}\right)$, we now use the fact that they agree to write

$$
(1-K) b_{0}\left(\underline{x}_{0}\right)+K \sum_{\underline{x}_{r_{i}} \backslash \underline{x}_{0}} b_{r_{i}}\left(\underline{x}_{i}\right)=\sum_{\underline{x}_{i} \backslash \underline{x}_{0}} b_{r_{i}}\left(\underline{x}_{i}\right),
$$

which concludes the induction base case with

$$
\forall_{i \in[1, \ldots, K]} b_{0}\left(\underline{x}_{0}\right)=\sum_{\underline{x}_{i} \backslash \underline{x}_{0}} b_{r_{i}}\left(\underline{x}_{i}\right)
$$

as desired.

Induction: Inductive step. Focus on a given region $r_{0}$, and consider its ancestry $A_{r_{0}}$. In a partial order, the ancestry of a given element is always generated by the union of ancestries of all elements covering it (see the previous appendix for the definition of the cover). Let there be $K$ such elements $r_{i}$ covering $r_{0}$, then

$$
A_{r_{0}}=\bigcup_{1 \leqslant i \leqslant K} A_{r_{i}}^{o}
$$

The induction step assumes that all ancestors $p \in A_{r_{i}}$ of $r_{i}$ are consistent with $r_{i}$, in the sense of (B2), and will then prove that $r_{i}$ has also to be consistent with $r_{0}$. Since consistency between any $p \in A_{r_{i}}$ and $r_{i}$ and between $r_{i}$ and $r_{0}$ is given, transitivity implies consistency between $p$ and $r_{0}$, and generalizing, with all the ancestry $A_{r_{0}}$ of $r_{0}$, concluding the induction step.

The tricky part is to show that consistency of the cover elements $r_{i}$ with their ancestry $A_{r_{i}}$ implies consistency of $r_{i}$ with $r_{0}$. In order to do so, let us start by the following:

Lemma 6 (Intersection of ancestries). If the set $\left\{r_{1}, \ldots, r_{K}\right\}$ covers the element $r_{0}$, then for any two distinct $k_{1}$ and $k_{2}$ in $[1, \ldots, K]$, if there exists $p \in A_{r_{k_{1}}}$ such that $\gamma=p \cap r_{k_{2}}>r_{0}$, then $\gamma=r_{k_{2}}$.

In other words, any element $p \in A_{r_{0}}$ such that the intersection $p \cap r_{i}$ with one of the covers $r_{i}$ is larger than $r_{0}$ is itself an ancestor of that cover $p \in A_{r_{i}}^{o}$.

Proof. It is enough to note that $\gamma=p \cap r_{k_{2}}>r_{0}$ is necessarily bounded $r_{0}<\gamma \leqslant r_{k_{2}}$, but since $r_{k_{2}}$ is a cover of $r_{0}$, no such intermediate element can exist, and therefore the only accepted situation is $\gamma=r_{k_{2}}$. But, this also implies that $r_{k_{2}}=p \cap r_{k_{2}}$ which means that $p$ is ancestor of $k_{2}$.

Corollary 4. The set $\partial m_{r_{i} \rightarrow r_{0}}=\left\{p \in A_{r_{0}}^{o} \mid p \cap r_{i}=r_{0}\right\}$ is given by

$$
\partial m_{r_{i} \rightarrow r_{0}}=A_{r_{0}}^{o} \backslash A_{r_{i}}^{o} .
$$

Graphically this means that the only possible situation for the sets $\partial m_{r_{i} \rightarrow r_{0}}$ is the one in the left panel of Fig. 7 and the situation in the right is forbidden.

Now, for every cover region $r_{i}, i \in[1, \ldots, K]$ we will have an Eq. (B1), that using the previous corollary can be written as

$$
\sum_{r \in A_{r_{0}}^{o}} c_{r} \sum_{\underline{x}_{r} \backslash \underline{x}_{0}} b_{r}\left(\underline{x}_{r}\right)=\sum_{r \in A_{r_{i}}^{o}} c_{r} \sum_{\underline{x}_{r} \backslash \underline{x}_{0}} b_{r}\left(\underline{x}_{r}\right) .
$$

Now, it is quite similar to the base case of the induction. The left hand side does not depend on $i \in[1, \ldots, K]$, while the 

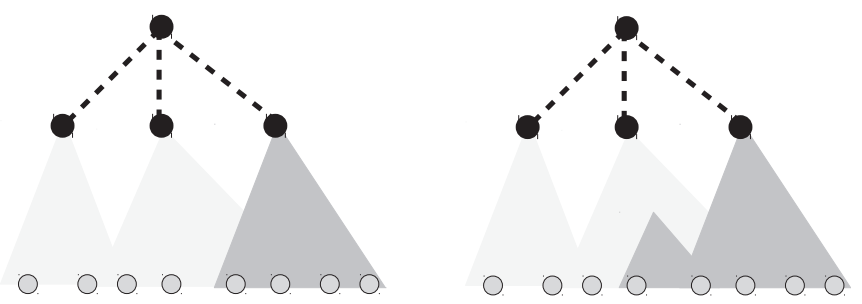

FIG. 7. Allowed (left) and not allowed (right) situations for the relation of $\partial m_{r_{i} \rightarrow r_{0}}$ with respect to the full ancestry of region $r_{0}$ (see Corollary 4). The message $m_{r_{i} \rightarrow r_{0}}$ appears in the belief equations of all the ancestors of region $r_{0}$ (light gray area), and $r_{0}$ itself, except for those who are ancestors of $r_{i}$ (dark gray area).

right hand side does. Therefore, any two $i_{1}, i_{2} \in[1, \ldots, K]$ will be consistent:

$$
\sum_{r \in A_{r_{i_{1}}}^{o}} c_{r} \sum_{\underline{x}_{r} \backslash \underline{x}_{0}} b_{r}\left(\underline{x}_{r}\right)=\sum_{r \in A_{r_{i_{2}}}^{o}} c_{r} \sum_{\underline{x}_{r} \backslash \underline{x}_{0}} b_{r}\left(\underline{x}_{r}\right) .
$$

Furthermore, using that $c_{r_{i}}=1-\sum_{r \in A_{r_{i}}} c_{r}$ and the consistency of $r_{i}$ with its ancestry, the previous equality can be transformed in

$$
\sum_{\underline{x}_{i_{1}} \backslash \underline{x}_{0}} b_{r_{i_{1}}}\left(\underline{x}_{r_{i_{1}}}\right)=\sum_{\underline{x}_{i_{2}} \backslash \underline{x}_{0}} b_{r_{i_{2}}}\left(\underline{x}_{r_{i_{2}}}\right) .
$$

Proceeding in a similar fashion as done in the base case for the induction, we can use the consistency between all different $r_{i}$ back in Eq. (B3) to show that they also have to agree with $r_{0}$ :

$$
b_{r_{0}}\left(\underline{x}_{r_{0}}\right)=\sum_{\underline{x}_{r_{i}} \backslash \underline{x}_{0}} b_{r_{i}}\left(\underline{x}_{r_{i}}\right),
$$

concluding the inductive step.

\section{APPENDIX C: MOMENT MATCHING FIELDS ARE GAUGE FREE}

Let us prove Theorems 4 and 5. They both say that using maximal messages with moment matching fields guarantees the consistency of beliefs, and is gauge free. We have already shown in the previous Appendix A that extremization of the CVM free energy with respect to message functions ensures consistency of the beliefs. However, when using moment matching fields, we reduce the degrees of freedom of the message functions, and therefore it is not clear that consistency still holds.

The reduction on the active fields was done seeking a gauge-free parametrization of the variational free energy. So, we would also like to prove that the solution to the extremization problem gives a unique solution to the fields defining the messages. In other words, when doing moment matching, we have removed all fields except those necessary to guarantee the consistency between the beliefs.

The proof of the consistency of the beliefs (Theorem 4) will be carried in the following way:

(1) Show that extremization with respect to moment matching fields on a set of variables $\prod_{i \in q} s_{i}$ ensure consistency of the corresponding moments $\left\langle\prod_{i \in q} s_{i}\right\rangle$ among those beliefs containing that group of variables.
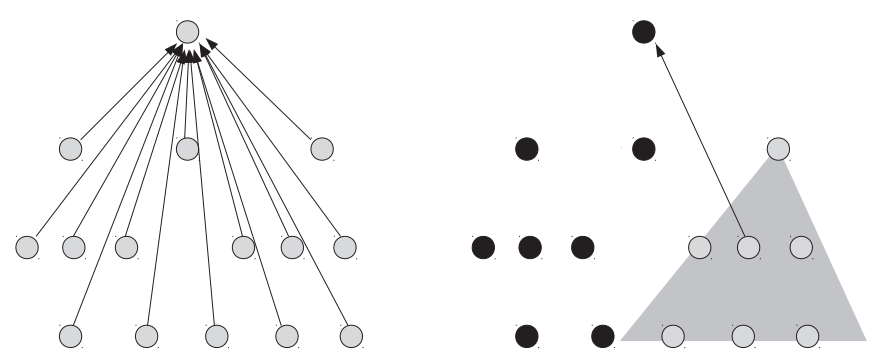

FIG. 8. Moment matching Definition 2. Right: all the ancestry of $r_{0}$ sends messages to this region. Left: if a particular type of field, let us say $U_{q}^{r_{i} \rightarrow r_{0}}$, is present in the message of one the covers $r_{i}$ of $r_{0}$, then that field is present in all the messages from the ancestry of $r_{i}$, marked in gray in the figure, and is not present in the rest of the messages coming from other ancestors. This can be rationalized as if the region $r_{i}$ and its ancestors are using a set of Lagrange multipliers $U_{q}^{p \rightarrow r_{0}}$ to coordinate their correlation among the variables $q$.

(2) Show that all moments are fixed by some field.

(3) Conclude by saying that if all moments are equal, distributions have to be consistent.

The proof of the gauge-free character (Theorem 5) will be carried out simply by showing that, after removing of the undesired fields, there are as many variables (fields) as equations to be solved.

\section{Moment consistency (Theorem 4)}

The prescription given by the moment matching Definition 2 says that message $m_{p \rightarrow r}\left(\underline{s}_{r}\right)$ counts with a field $U_{q}$ forcing the correlation among variables in $q \subseteq r$ as

$$
m_{p \rightarrow r}\left(\underline{s}_{r}\right)=e^{\cdots+U_{q}^{p \rightarrow r}} \prod_{i \in q} s_{i}+\cdots
$$

if and only if $r$ is the smallest region containing the subset $q$. Therefore, for any given set $q=\left\{s_{q_{1}}, \ldots, s_{q_{k}}\right\}$ there is a single region $r_{0}$ such that the fields $U_{q}^{p \rightarrow r_{0}}$ appear in the message from its ancestors $p \in A_{r_{0}}$. The situation is depicted in Fig. 8 .

When extremizing with respect to the fields $U$ that parametrize the messages, we get equations similar to (B1), but instead of the belief functions, we get the moments corresponding to the field

$$
\begin{aligned}
\forall_{p \in A_{r_{0}}^{o}} \sum_{r \in \partial U_{q}^{p \rightarrow r}} c_{r} \xi_{q, r} & =0 \\
\text { with } \quad \xi_{q, r} & =\left\langle\prod_{i \in q} x_{i}\right\rangle_{b_{r}}
\end{aligned}
$$

and the expected value is taken with respect of the belief $b_{r}\left(\underline{x}_{r}\right)$ (local distribution) at region $r$ and $\partial U_{q}^{p \rightarrow r}=\partial m_{p \rightarrow r}$ is the set of regions in whose beliefs the message $m_{p \rightarrow r}$ participates and, therefore, so does $U_{q}^{p \rightarrow r}$.

We will have as many equations (C1) as ancestors $r_{0}$ have $K=\left|A_{r_{0}}\right|$, corresponding to derivation with respect to each field (each arrow in Fig. 8). We will further assume that no counting number is zero, and therefore the set of linear equations (C1) relates all moments $\xi_{q, r}$ which are $K+1$, including the one obtained at $r_{0}$ itself. 
Since we have proved in Appendix A that

$$
\sum_{r \in \partial U_{q}^{p \rightarrow r}} c_{r}=0
$$

a particular solution of the system of equations will always be

$$
\forall_{r \in A_{r_{0}}} \xi_{q, r}=\xi_{q, r_{0}},
$$

which is part of what we are trying to prove. We can also write Eq. (C1) as

$$
\forall_{p \in A_{r_{0}}} \sum_{r \in \partial U_{q}^{p \rightarrow r} \backslash r_{0}} c_{r} \xi_{q, r}=-c_{r_{0}} \xi_{q, r_{0}},
$$

where the right hand side is the same irrespective of who is $p$. Considering only the correlations involved in the left hand side, this system of equations will have only one solution (at fixed $\xi_{q, r_{0}}$ ) if the matrix $G_{K \times K}$ made of elements

$$
g_{r, p}=\left\{\begin{array}{cc}
c_{r} & \text { if } r \in \partial U_{q}^{p \rightarrow r_{0}} \\
0 & \text { otherwise }
\end{array}\right.
$$

has nonzero determinant.

In order not to make the paper far too long, we ask the reader to prove in each cluster variational method implemented that the set of regions used fulfill this property. Yet, we warn that this property will not be fulfilled any time that some of the following conditions are present:

(i) There are zero counting numbers since a column of the matrix will be full of zeros.

(ii) A given message does not appear in any belief equation since a row will be full of zeros.

(iii) Two or more rows of the matrix are equal, causing a zero determinant.

Without a proof (that seems rather complicated to us), we give the hint that these seem to be the only situations possible, after many random playing with arbitrary cluster approximations. It is not obvious why two or more lines could not be linearly combined into another line, to cause a zero determinant, but some properties of the counting numbers seem to forbid this.

So, we have that under the condition of nonsingular matrix $G_{K \times K}$ the set of equations involving the correlation of a given set of spins $q$ force all such correlations to be equal. Now, since every set of spins contained in two or more regions is contained in their intersection (which also has to be a region by CVM prescription), then all two regions agree on the moments of every common subset of variables.

We finish the proof by noting that if all regions agree on all correlations of the intersecting variables, they have to be consistent in the sense that the marginal probabilities over these variables should agree.

\section{Gauge free (Theorem 5)}

We note from the previous proof that we have $K$ fields $U_{q}^{p \rightarrow r_{0}}$ if there are $K$ ancestors of region $r_{0}$. Since the consistency of the corresponding correlations $\xi_{q, p}$ is fixed by $K$ equations, we have as many parameters as equations to be satisfied. Furthermore, the consistency among the local distributions $b_{r}\left(\underline{x}_{r}\right)$ that contain a given set of variables $q$ can not be forced with less than $K$ equalities since equalities are transitive and, therefore, all we need is to connect the set of $K+1$ regions containing $q$ in a graph with minimal number of edges (each edge meaning an equality) among moments. Among $K+1$ nodes, the single component graph with minimal edges is the tree, which happens to have $K$ edges. So, as we said, the $K$ fields $U_{q}^{p \rightarrow r_{0}}$ are exactly the minimum required amount to enforce all local distributions to agree on the respective moment $\xi_{q, p}$.

This was not the case in parent-to-child CVM, for instance, as seen in [19], where the consistency of a single spin magnetization that belonged to four links and four plaquettes in the square plaquette Ising model, appear after the derivation with respect to 12 parameters (field) instead of 8 . Therefore, there are 12 equations (and 12 parameters) to assign the equality among 8 local distributions, forcing 4 of the equations (and parameters) to be redundant.

So, in this appendix we have proved that maximal messages and moment matching fields generate a set of equations with the following properties:

(1) Every correlation among a set of variables that belongs to two or more regions is present in some equation.

(2) There are as many free parameters as relations required to guarantee consistency.

(3) Consistent correlations are one solution of the system.
[1] K. Huang, Statistical Mechanics (Wiley, New York, 1987).

[2] G. Parisi, M. Mézard, and M. Virasoro, Spin Glass Theory and Beyond (World Scientific, Singapore, 1987).

[3] M. Mézard and G. Parisi, Eur. Phys. J. B 20, 217 (2001).

[4] M. Mézard and G. Parisi, J. Stat. Phys. 111, 1 (2003).

[5] M. Mézard and A. Montanari, Information, Physics, and Computation (Oxford University Press, New York, 2009).

[6] J. Yedidia, W. T. Freeman, and Y. Weiss, IEEE Trans. Inf. Theory 51, 2282 (2005).

[7] T. Rizzo, A. Lage-Castellanos, R. Mulet, and F. Ricci-Tersenghi, J. Stat. Phys. 139, 375 (2010).

[8] A. Lage-Castellanos, R. Mulet, F. Ricci-Tersenghi, and T. Rizzo, J. Phys. A: Math. Theor. 46, 135001 (2013).
[9] J.-Q. Xiao and H. Zhou, J. Phys. A: Math. Theor. 44, 425001 (2011).

[10] H. Zhou and C. Wang, J. Stat. Phys. 148, 513 (2012).

[11] E. Domínguez, A. Lage-Castellanos, and R. Mulet, J. Stat. Mech.: Theor. Exp. (2015) P07003.

[12] K. Tanaka and T. Morita, Phys. Lett. A 203, 122 (1995).

[13] K. Tanaka, J. Inoue, and D. M. Titterington, in XIII Proceedings of the 2003 IEEE Signal Processing Society Workshop, 17-19 September, 2003 (IEEE Computer Society, Piscataway, NJ, 2003), pp. 329-338.

[14] M. Yasuda, S. Kataoka, and K. Tanaka, Phys. Rev. E 92, 042120 (2015). 
[15] E. Gouillart, F. Krzakala, M. Mézard, and L. Zdeborová, Inverse Probl. 29, 035003 (2013).

[16] J.-C. Sibel and S. Reynal, International Conference on Control, Automation and Information Sciences (IEEE, Piscataway, NJ, 2012), p. 4.

[17] P. Pakzad and V. Anantharam, Neural Comput. 17, 1836 (2005).

[18] C. Wang and H.-J. Zhou, J. Phys.: Conf. Ser. 473, 012004 (2013).

[19] E. Domínguez, A. Lage-Castellanos, R. Mulet, F. RicciTersenghi, and T. Rizzo, J. Stat. Mech.: Theor. Exp. (2011) P12007.

[20] C. Furtlehner and A. Decelle, J. Stat. Phys. 164, 531 (2016).

[21] M. Opper and O. Winther, J. Machine Learning Res. 6, 2177 (2005).

[22] M. Yasuda, S. Kataoka, and K. Tanaka, J. Phys. Soc. Jpn. 81, 044801 (2012).
[23] A. Montanari and T. Rizzo, J. Stat. Mech.: Theor. Exp. (2005) P10011.

[24] J. S. Yedidia, in Advanced Mean Field Methods: Theory and Practice, edited by D. Saad and M. Opper (MIT Press, Cambridge, MA, 2000), Chap. 3, p. 21.

[25] R. Kikuchi, Phys. Rev. 81, 988 (1951).

[26] L. Onsager, Phys. Rev. 65, 117 (1944).

[27] A. Lage-Castellanos, R. Mulet, F. Ricci-Tersenghi, and T. Rizzo, Phys. Rev. E 84, 046706 (2011).

[28] M. N. Barber, R. B. Pearson, D. Toussaint, and J. L. Richardson, Phys. Rev. B 32, 1720 (1985).

[29] D. Gaunt, M. Sykes, and S. McKenzie, J. Phys. A: Math. Gen. 12, 871 (1979).

[30] G. Parisi and J. J. Ruiz-Lorenzo, Phys. Rev. B 54, R3698 (1996). 